\title{
Nuevos diseños de filtros planares en tecnologías de microcinta y finline utilizando resonadores de anillos divididos
}

\author{
Novel designs of planar filters in microstrip and \\ finline technologies using split ring resonators
}
A. León ${ }^{1}$
A. Casanueva ${ }^{2}$
J. Herrero ${ }^{2}$
F. Marante ${ }^{1}$

Recibido 30 de noviembre de 2010, aceptado 9 de noviembre de 2012

Received: November 30, $2010 \quad$ Accepted: November 9, 2012

\begin{abstract}
RESUMEN
En este artículo se presenta una técnica mejorada para diseñar filtros en configuraciones planares. Este nuevo procedimiento se aplica para mejorar el desempeño de estos dispositivos. Este trabajo ilustra que los resonadores de anillos complementarios divididos (CSRRs) acoplados eléctricamente a una línea de transmisión y los resonadores de anillos divididos (SRRs) acoplados magnéticamente a una estructura finline pueden emplearse con la intención de mejorar el diseño de tales filtros. Con este propósito se analizan variantes clásicas de filtros de ondas milimétricas. Estos componentes incluyen filtros pasabajo de microcinta, filtros finline pasabanda y supresores de banda, así como filtros supresores de banda en finline antipodal. Se discuten las propiedades de estas configuraciones y los resultados experimentales se encuentran en buen acuerdo con las simulaciones. Los resultados muestran la utilidad y validez de las nuevas estructuras ya que es posible lograr bajas pérdidas de inserción y bandas de transición muy agudas con alta capacidad de rechazo.
\end{abstract}

Palabras clave: Filtros planares, finline, finline antipodal, resonadores de anillos divididos, taper.

\begin{abstract}
In this paper an improved technique is outlined to design filters in planar configurations. The new procedure is applied to enhance the performance of these devices. This work illustrates that complementary splitring resonators (CSRRs), electrically coupled to a microstrip transmission line and split-ring resonators (SRRs) magnetically coupled to a finline structure can be applied to improve the design of such filters. For this purpose, classical variants of millimeter wave filters have been analyzed. These components include microstrip low-pass filters, finline bandpass/bandstop filters and antipodal finline bandstop filters. The properties of these configurations are discussed; the experimental data are in good agreement with the simulations. The results show the usefulness and validity of the new structures as it is possible to achieve a very low insertion loss and very sharp transition bands with high rejection capabilities.
\end{abstract}

Keywords: Planar filters, finline, antipodal finline, split-ring resonators, taper.

\section{INTRODUCCIÓN}

En la actualidad se requiere que los circuitos que operan en las bandas de microondas y milimétricas sean enormemente miniaturizados y que a la vez presenten un buen desempeño. Uno de los campos de investigación más actualizados es el filtrado de ciertas bandas de frecuencia para la supresión de los espurios de una señal y los armónicos indeseados en los circuitos de microondas [1-4].

1 Departamento de Telecomunicaciones y Telemática. Instituto Superior Politécnico José Antonio Echeverría. Ave 114 No 11901 Código postal 19390. C. Habana, Cuba. E-mail: alainleon@electrica.cujae.edu.cu; marante@electrica.cujae.edu.cu

2 Departamento de Ingeniería de Comunicaciones. Universidad de Cantabria. Avda Los Castros, s/n - 39005. Santander, España. E-mail: alicia.casanueva@unican.es 
Los resonadores de anillos divididos (SRRs) son estructuras compactas con parámetros fácilmente escalables que proveen una alternativa poderosa a las estructuras EBG (Electromagnetic band gap) que han sido estudiadas por varios años [5-9]. Los resonadores de anillos divididos (SRRs) junto con los resonadores de anillos complementarios divididos (CSRRs) contribuyen a mantener el tamaño pequeño del filtro pasabanda, dado que su dimensión es inferior a la longitud de onda, permitiendo así un agudo rechazo [10-18]. Aunque se han desarrollado variedades de configuraciones de filtros para reducir el nivel de los armónicos de las señales, en este trabajo se diseña un filtro pasabajo con paso de impedancia (SILPF) utilizando estructuras asimétricas de pasos de microcinta. Se propone además la incorporación de CSRRs. Al incorporar los CSRRs se demuestra que es factible producir un filtro que funcione sin rizado en la banda de paso a la misma vez que produce un agudo rechazo de la banda suprimida en la vecindad de su frecuencia de resonancia. Este artículo expande el procedimiento anterior al diseño de filtros de tipo finline. Los SRRs son resonadores de dimensiones inferiores a la longitud de onda que son capaces de inhibir la propagación de una banda estrecha de frecuencias en la vecindad de su frecuencia de resonancia. Dado que el campo magnético se polariza a lo largo del eje de los anillos y que las estructuras finline presentan una distribución del campo magnético apropiada, esta tecnología finline se ha establecido como un acercamiento práctico en el diseño y construcción de componente y subsistemas de ondas milimétricas. En años recientes se han analizado ampliamente varios filtros finline [19-20]. Sin embargo no se ha podido proveer de una amplia banda de rechazo con alto nivel de atenuación. Dado que los modos de órdenes superiores ocurren entre las discontinuidades, es fundamental para el funcionamiento apropiado del filtro reducir estas discontinuidades. Con este fin, este artículo apunta a encontrar alternativas a los filtros convencionales, principalmente en las discontinuidades de la ranura de la finline. Considerando que entre las diferentes estructuras de finline la estructura de línea antipodal provee un amplio ancho de banda [21], se ha diseñado un filtro supresor de banda (BSF) con una banda suprimida extremadamente ancha.

Este trabajo ha sido organizado de la siguiente manera. Inicialmente se optimiza el SILPF en tecnología de microcinta utilizando los CSRRs. Una vez que se alcanzan los dos objetivos de reducir el tamaño del circuito y de empujar significativamente los espurios a frecuencias más elevadas, el próximo paso es mejorar los resultados anteriores. Con este fin, un nuevo modelo de SILPF y su estructura complementaria (CSRRs) se presentan y analizan. Los resultados medidos del filtro prototipo se encuentran en buen acuerdo con los resultados simulados [22] y las respuestas del filtro prototipo muestran una amplia banda suprimida con alta atenuación, alta selectividad y un pequeño nivel de rizado en la banda pasante. Esto se atribuye a los resonadores grabados en el plano tierra. Luego se utilizan los SRRs en el diseño de filtros finline tipo pasabanda y supresores de banda (BPF/BSF). Se proponen un filtro pasabanda en estructura finline y uno supresor de banda en estructura finline antipodal. El proceso de análisis es muy similar al de tecnología de microcinta. Después de que se obtuvieron los resultados, se pensó que estas estructuras basadas en SRRs son de interés para el diseño de filtros planares. Los resultados obtenidos utilizando un conjunto de anillos resonantes sugieren que estos elementos y sus duales proveen herramientas que mejoran drásticamente la respuesta de los filtros en tecnología planar. Los resultados teóricos y experimentales se obtuvieron utilizando el simulador electromagnético de ANSOFT de onda completa HFSS y el analizador de redes HP8510.

\section{ANÁLISIS TEÓRICO}

\section{Configuración de microcinta utilizando CSRRs}

El propósito de esta sección es extender el análisis al diseño de un filtro pasabajo de microcinta utilizando dos parejas de resonadores de anillos complementarios divididos orientados un ángulo arbitrario $\theta$ [22]. El objetivo principal aquí es ver cómo el funcionamiento de un filtro pasabajo mejora en tecnología de microcinta al incorporar resonadores de anillos complementarios divididos. La Figura 1 representa la topología del filtro pasabajo de microcinta.

Al grabar los CSRRs en el plano tierra y debajo del SILPF puede asegurarse que la partícula se excite apropiadamente. Esto se logra a través de un campo eléctrico polarizado en la dirección axial de los anillos. Alrededor de la frecuencia de resonancia se introduce una profunda banda de rechazo debido a la 


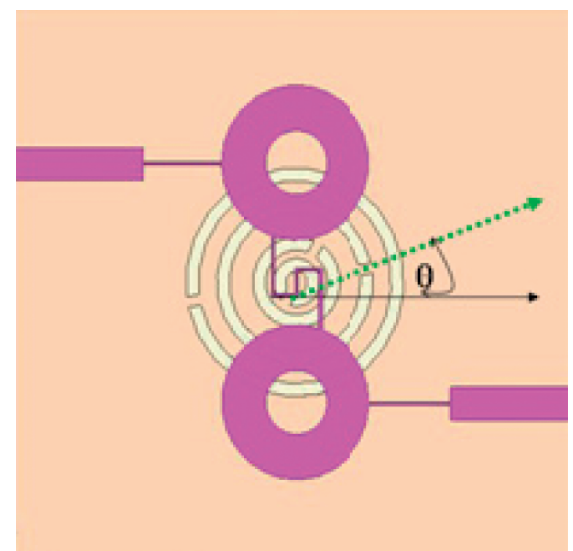

Figura 1. Vista esquemática del filtro pasabajo de microcinta propuesto.

presencia de una permitividad efectiva negativa. Un CSRR es la imagen negativa de un SRR y produce una banda suprimida de alto rechazo en la vecindad de su frecuencia de resonancia. El comportamiento correcto de la célula CSRR depende básicamente de tres factores: la geometría, el dieléctrico y la posición del CSRR en el plano tierra del SILPF. Primeramente se diseñó el CSRR. Las dimensiones se escogieron de tal manera que la primera frecuencia de resonancia del CSRR fuera igual a la frecuencia de corte de -3 $\mathrm{dB}$ del SILPF que se requería que fuera de $3 \mathrm{GHz}$. El diseño y cálculo de la frecuencia de resonancia del CSRR no se discute aquí ya que una explicación detallada se brinda en [13].

\section{Filtros finline utilizando SRRs}

En este artículo se presenta una nueva técnica para desarrollar un filtro finline pasabanda (BPF) y un filtro finline antipodal supresor de banda (BSF). Las Figuras $8 \mathrm{a}$ y $8 \mathrm{~b}$ muestran las configuraciones unilateral y antipodal, respectivamente.

El mismo procedimiento de diseño que se aplicó en el filtro pasabajo de microcinta puede aplicarse para diseñar un filtro BPF/BSF en configuración finline utilizando SRRs. En este artículo se mostrará que pueden utilizarse como elementos los SRRs tomados en parejas para mejorar la respuesta de los filtros de estas configuraciones. Para este propósito, primeramente se utiliza el método convencional de filtro pasabanda. Una vez que se encuentra la respuesta electromagnética simulada, una célula de SRR se coloca en la estructura del filtro finline como se muestra en la Figura 9.

\section{RESULTADOS}

La Tabla 1 y la Figura 2 muestran el diseño típico de los parámetros geométricos. Una vez que la estructura clásica del filtro ha sido validada, ver Figura 5, el próximo paso es colocar apropiadamente el CSRR adecuado para mejorar la respuesta del filtro anterior.

Tabla 1. Dimensiones del doble CSRR.

\begin{tabular}{|c|c|}
\hline Parámetros & Dimensiones $(\mathbf{m m})$ \\
\hline $\mathrm{r} 1$ & 0,41051 \\
$\mathrm{r} 2$ & 1,21051 \\
$\mathrm{r} 3$ & 2,6 \\
$\mathrm{r} 4$ & 2,6 \\
\hline $\mathrm{c}$ & 0,4 \\
\hline $\mathrm{d}$ & 0,6 \\
\hline $\mathrm{g}$ & 0,4 \\
\hline Sustrato & $\varepsilon_{\mathrm{r}}=3,2,(\delta=0,003)$ \\
$(\mathrm{L} \times \mathrm{W} \times \mathrm{H})$ & $22,19 \times 20,926 \times 0,51$ \\
\hline
\end{tabular}

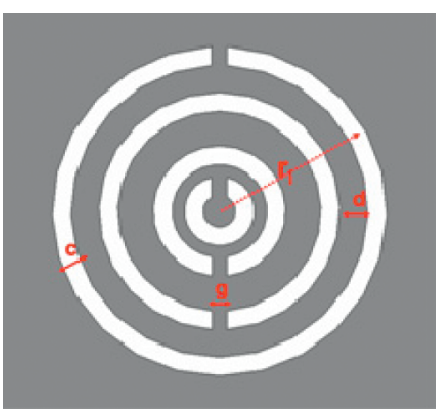

Figura 2. Geometría de la célula unitaria del CSRR.

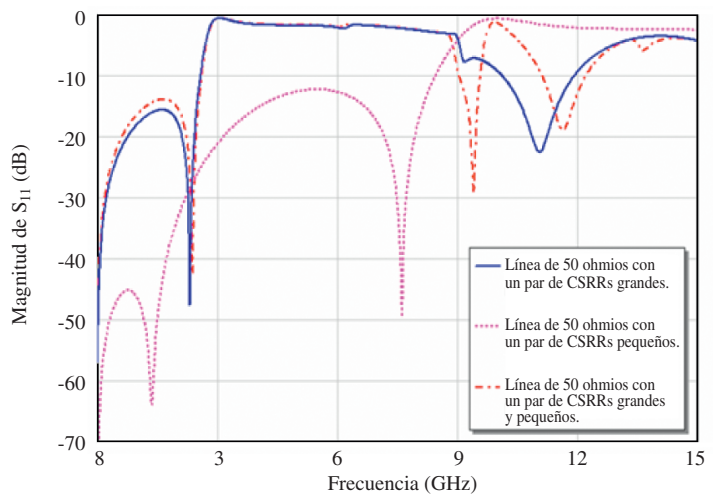

Figura 3. Línea de microcinta de $50 \mathrm{Ohms}$ cargada con CSRR. 

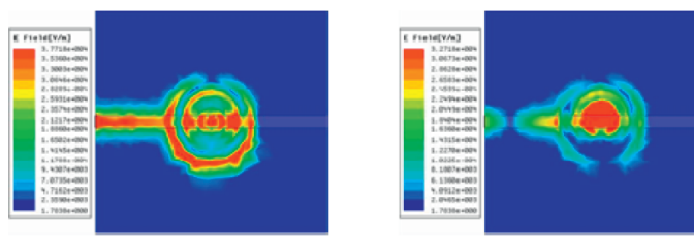

Frat resonant frequency: $2.046 \mathrm{CH}$
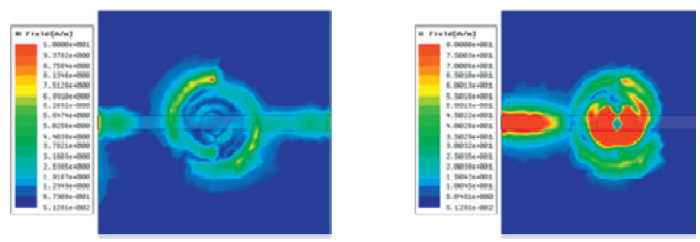

Figura 4. Distribución del campo electromagnético para las dos primeras frecuencias de resonancia.

Las Figuras 3 y 4 muestran la respuesta de frecuencia típica de los CSRRs y su distribución de campo electromagnético respectivamente. Una vez que se ha visto como la frecuencia de resonancia del CSRR puede sintonizarse con la ayuda de las dimensiones del dispositivo, el segundo paso es colocar esta estructura en la posición correcta del plano tierra del filtro. Este filtro presenta propiedades especiales: es posible variar los parámetros de dispersión modificando el radio tanto de los resonadores de la pared superior como los de la inferior. Esta flexibilidad es una propiedad muy importante si se desea la optimización del filtro. La Figura 5 muestra el esquema superior del filtro y la Tabla 2 las dimensiones geométricas.

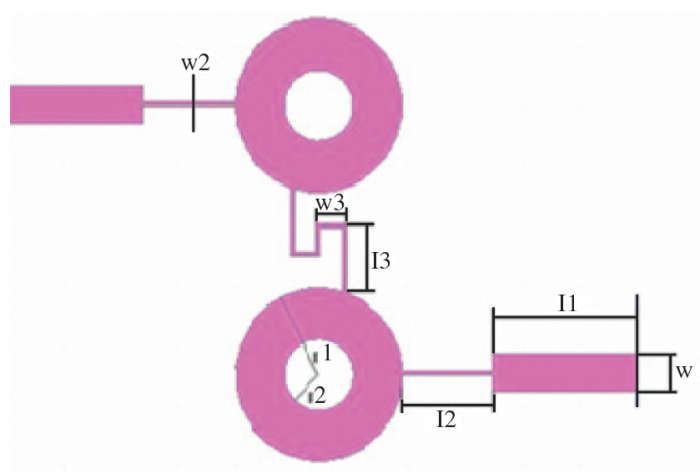

Figura 5. Esquema superior.

La Figura 6 ilustra que este filtro produce una banda suprimida con agudo rechazo en la vecindad de su frecuencia de resonancia y las pérdidas de retorno dependen de la orientación de los CSRRs, lográndose una respuesta muy plana en la banda suprimida cuando los resonadores se rotan en un ángulo $\theta=90^{\circ}$.
Tabla 2. Dimensiones del SILPF.

\begin{tabular}{|c|c|}
\hline Parámetros & Dimensiones $(\mathbf{m m})$ \\
\hline $\mathrm{r}_{1}$ & 2,9 \\
$\mathrm{r}_{2}$ & 1,205 \\
\hline $\mathrm{l}_{1}$ & 5,0 \\
$\mathrm{l}_{2}$ & 3,19 \\
$\mathrm{l}_{3}$ & 2,276 \\
\hline $\mathrm{w}_{1}$ & 1,2266 \\
$\mathrm{w}_{2}$ & 0,14 \\
$\mathrm{w}_{3}$ & 1,034 \\
\hline
\end{tabular}

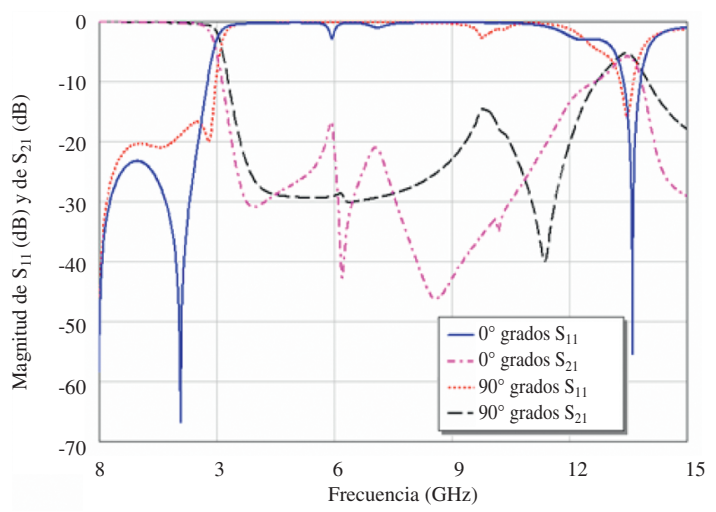

Figura 6. Magnitud de los parámetros de dispersión contra frecuencia para dos orientaciones diferentes de los CSRRs.

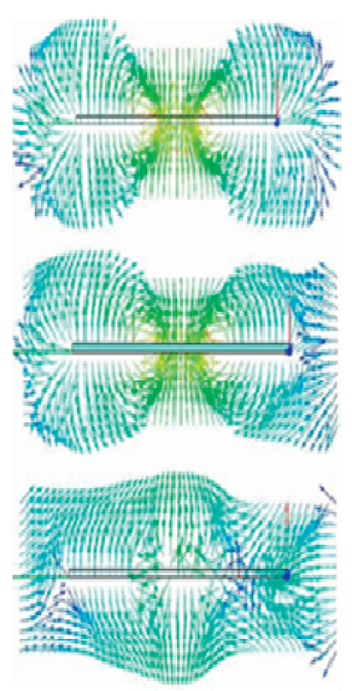

(a)

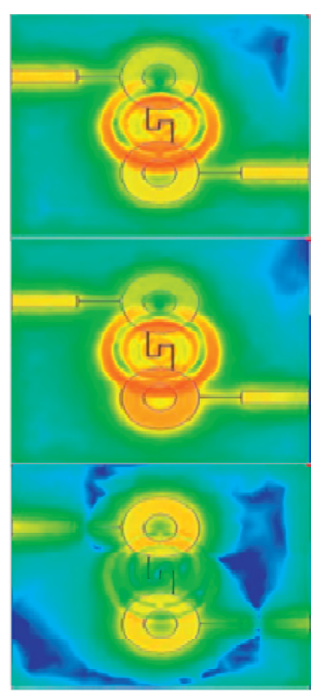

(b)
Figura 7. Distribución del campo eléctrico. a) Sección transversal, b) Vista frontal de la cara inferior del sustrato. 
En la Figura 7 es posible observar cómo la orientación y concentración del campo eléctrico se modifican de acuerdo a la operación de la frecuencia: a) antes de la frecuencia de resonancia $2,93 \mathrm{GHz}$, b) en la frecuencia de resonancia del CSRR $3,08 \mathrm{GHz}$ y c) después de la frecuencia de resonancia 3,23 GHz.

Esta sección discute varios filtros en estas dos configuraciones. Aquí, debido a las distribuciones del campo magnético en las estructuras finline, los SRRs se excitan en una forma más efectiva.

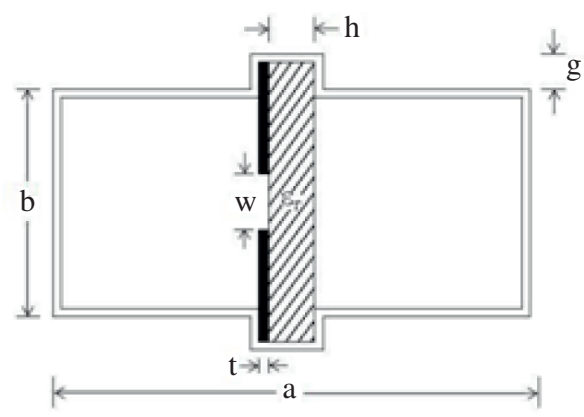

(a)

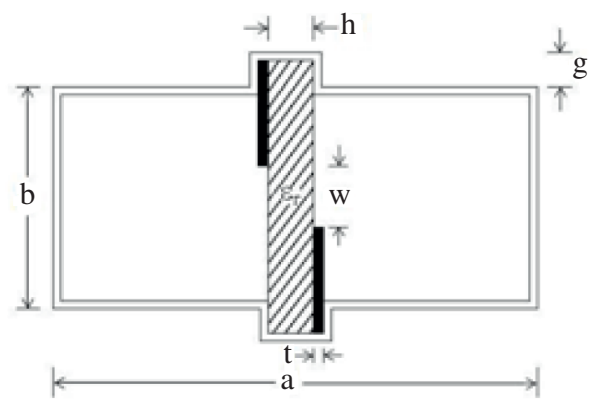

(b)

Figura 8. Estructuras finline: a) Finline unilateral, b) Finline antipodal.

La estructura propuesta muestra una ancha banda suprimida con alta atenuación, alta selectividad y un pequeño nivel de rizado en la banda pasante, lo cual se atribuye a los dobles resonadores de anillo. Después de eso, el resonador se colocó en la cara inferior del sustrato y la respuesta es similar, tal como ilustra la Figura 9c. Las Tablas 3 y 4 presentan los parámetros geométricos y eléctricos.
Tabla 3. Dimensiones de los resonadores de anillos divididos (SRRs).

\begin{tabular}{|c|c|}
\hline Parámetros & Dimensiones (mm) \\
\hline $\mathrm{r}$ & 2,425 \\
\hline $\mathrm{c}$ & 0,533 \\
\hline $\mathrm{d}$ & 0,435 \\
\hline $\mathrm{g}$ & 0,4 \\
\hline
\end{tabular}

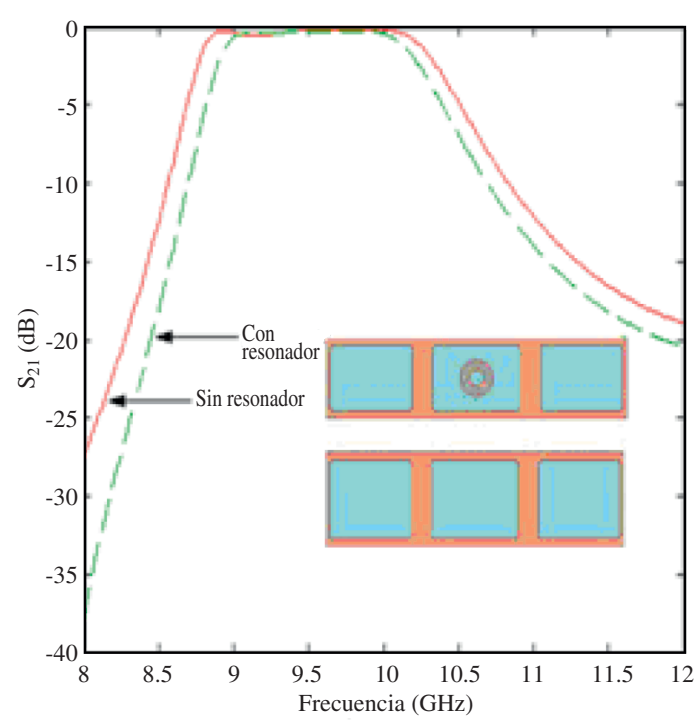

(a)

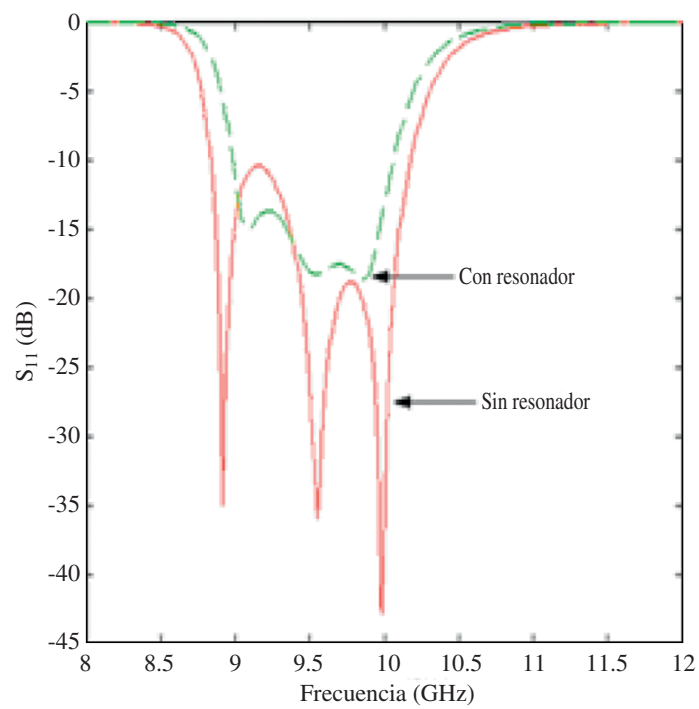

(b) 

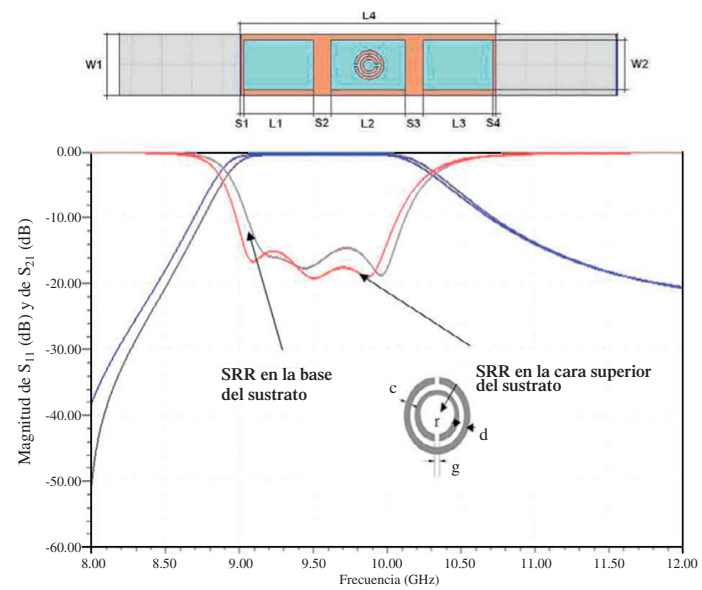

(c)

Figura 9. Pérdidas de transmisión y esquema: a) Filtro finline plano-E en la guía WR90 cargada con SRRs, b) Pérdidas de retorno, c) Pérdidas de transmisión para diferentes posiciones de los SRRs.

El próximo paso en este trabajo involucra el diseño de dos nuevos prototipos de BSF que utilizan SRRs en configuración finline y antipodal. El primero se diseñó utilizando 5 células de SRR. La Figura 10a ilustra los parámetros de transmisión en función
Tabla 4. Dimensiones del filtro finline pasabanda.

\begin{tabular}{|c|c|}
\hline Parámetros & Dimensiones (mm) \\
\hline Ancho de la guía, $a$ & 22,86 \\
Ancho del sustrato, $w_{1}$ & 10,16 \\
Ancho de la ranura, $w_{2}$ & 8,16 \\
\hline $\begin{array}{c}\text { Longitud total del filtro de } \\
\text { guía de ondas, } L 4 \\
\text { Ancho de la metalización, } \mathrm{t}\end{array}$ & 42,058 \\
\hline Longitud de la unidad & 0,035 \\
resonadora, & \\
L1 & 11,466 \\
L2 & 12,216 \\
L3 & 11,466 \\
\hline Longitud del septum & \\
metálico & \\
S1 & 0,493 \\
S2 & 2,962 \\
S3 & 2,962 \\
S4 & 0,493 \\
\hline Sustrato & $\varepsilon_{\mathrm{r}}=3,2,(\delta=0,003)$ \\
(L x W x H) & $42,058 \times 8,16 \times 1,56$ \\
\hline \multicolumn{2}{|c}{} \\
\end{tabular}

de la frecuencia junto con la geometría del filtro, y las Figuras 10b y 10c muestran los campos eléctrico y magnético en dos frecuencias diferentes cercanas a la banda suprimida y a la banda pasante respectivamente.

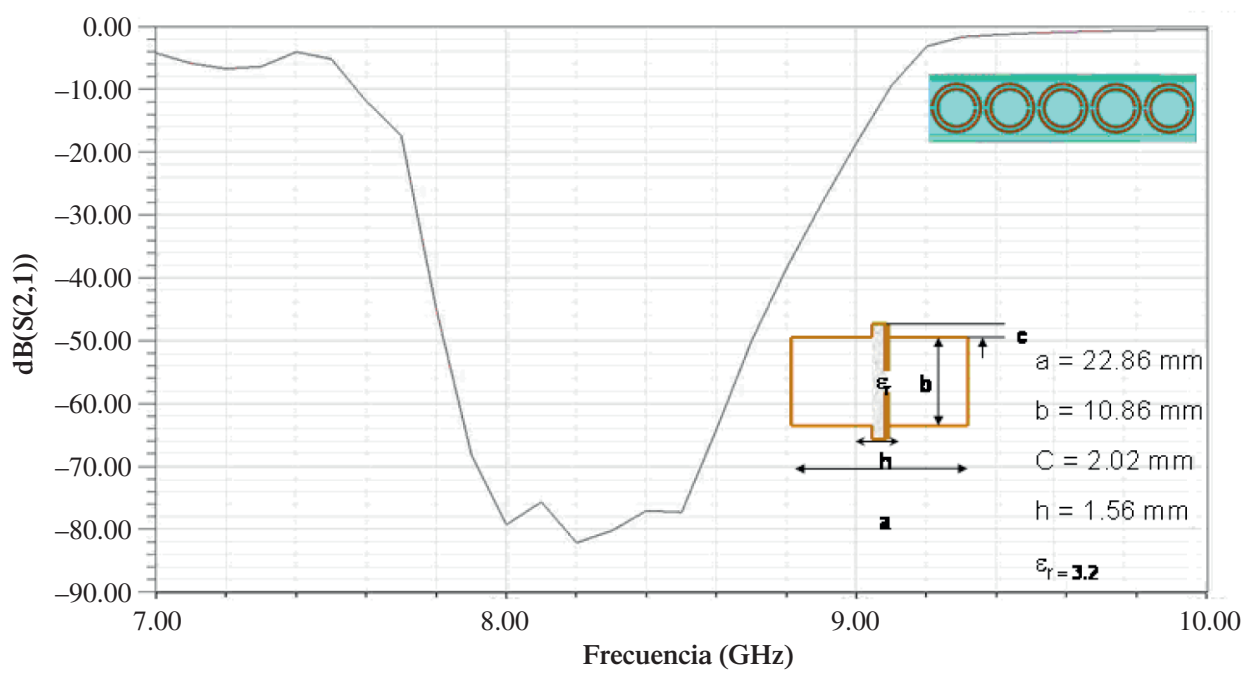

Figura 10a. Pérdidas de inserción del BSF en configuración finline y su esquema. 


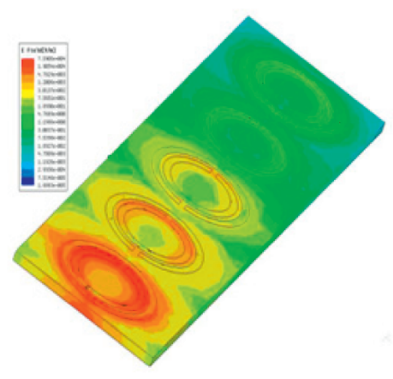

(a)

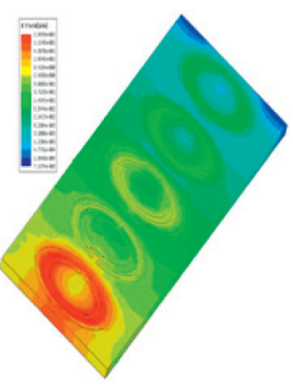

(b)
Figura 10b. Distribución del campo (a) eléctrico (b) magnético en la banda suprimida (8.7 GHz).

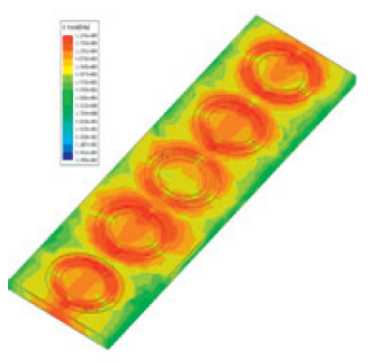

(a)

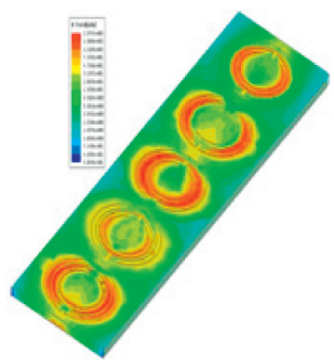

(b)
Figura 10c. Distribución del campo (a) eléctrico (b) magnético en la banda pasante (12 GHz).

El segundo prototipo BSF se construyó utilizando 4 células de SRR en cada cara del sustrato de la finline de la guía de ondas. La Figura 11 muestra este filtro. La función utilizada para describir el taper de la finline sigue la siguiente distribución seno cuadrado:

$$
W(z)=[(b+w)] \times \operatorname{sen}^{2}(\pi z / 2 L)
$$

donde $\mathrm{b}$, w y L son constantes que se ajustan numéricamente con el propósito de lograr la altura deseada del taper.

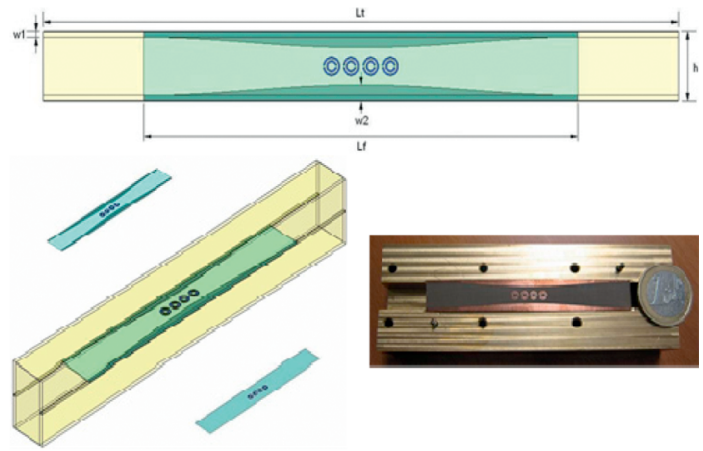

Figura 11. BSF en estructura finline.
Las Tablas 5 y 6 presentan los parámetros geométricos y eléctricos.

Tabla 5. Dimensiones de los resonadores de anillos divididos (SRRs).

\begin{tabular}{|c|c|}
\hline Parámetros & Dimensiones (mm) \\
\hline $\mathrm{r}$ & 1,55 \\
\hline $\mathrm{c}$ & 0,3 \\
\hline $\mathrm{d}$ & 0,3 \\
\hline $\mathrm{g}$ & 0,3 \\
\hline
\end{tabular}

La Figura 12 presenta los excelentes resultados obtenidos, así como el buen acuerdo que existe entre los resultados simulados y medidos, tanto en fase como en los parámetros de dispersión. El ligero desplazamiento existente entre la respuesta simulada y la medida posiblemente es una consecuencia del uso del valor de permitividad proporcionado por el fabricante del sustrato y a las estrictas tolerancias existentes durante el proceso de fabricación del circuito.

Tabla 6. Dimensiones del filtro finline supresor de banda.

\begin{tabular}{|c|c|}
\hline Parámetros & $\begin{array}{c}\text { Dimensiones } \\
(\mathbf{m m})\end{array}$ \\
\hline Ancho de la guía, a & 19 \\
Altura de la guía (con & 11,2515 \\
ranura) h & 9,5 \\
Altura de la guía (sin ranura) & \\
\hline Longitud total, LT & 120 \\
Longitud del filtro, LF & 82,097 \\
Ancho del filtro, $\mathrm{h}$ & 11,2512 \\
Ancho de la tira de metal y & 0,8756 \\
de la ranura, w1 & 2,5502 \\
Ancho de la tira de metal, & 0,0017 \\
w2 & 0,6 \\
Ancho de la metalización, t & \\
Separación entre & \\
resonadores, d & 82,097 x 11,2512 \\
& x 0,254 \\
Sustrato & Arlon CuClad 217 \\
(L x W x H) & $\varepsilon_{\mathrm{r}}=2,17$ \\
\hline
\end{tabular}




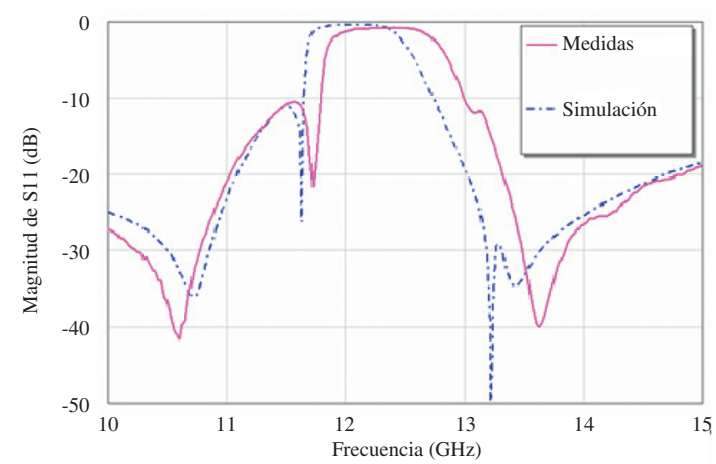

(a)

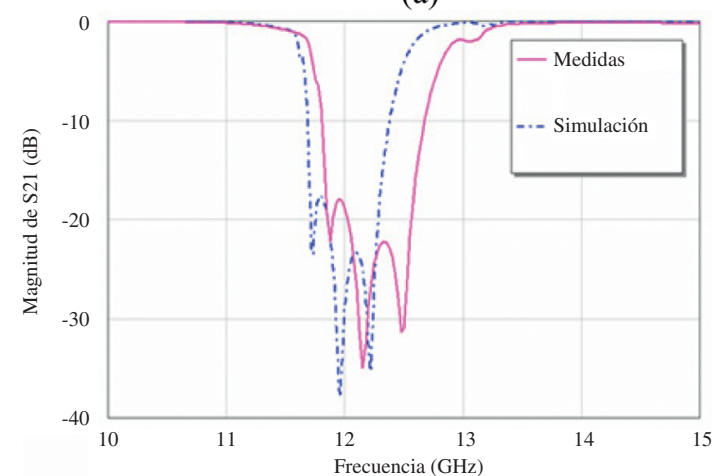

(b)

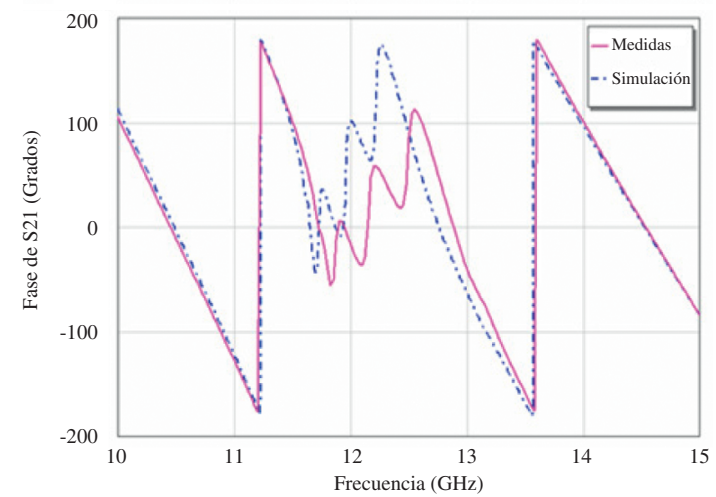

(c)

Figura 12. Parámetros de dispersión medidos y simulados contra frecuencia. (a) Magnitud de las pérdidas de retorno; (b) Magnitud de las pérdidas de inserción; (c) Fase de las pérdidas de inserción contra frecuencia.

Dados los buenos resultados obtenidos anteriormente, se decidió extender la configuración anterior al diseño de un filtro en configuración antipodal para observar cómo al variar las dimensiones de los resonadores se puede desplazar la banda del filtro, ya que la configuración antipodal presenta un mayor ancho

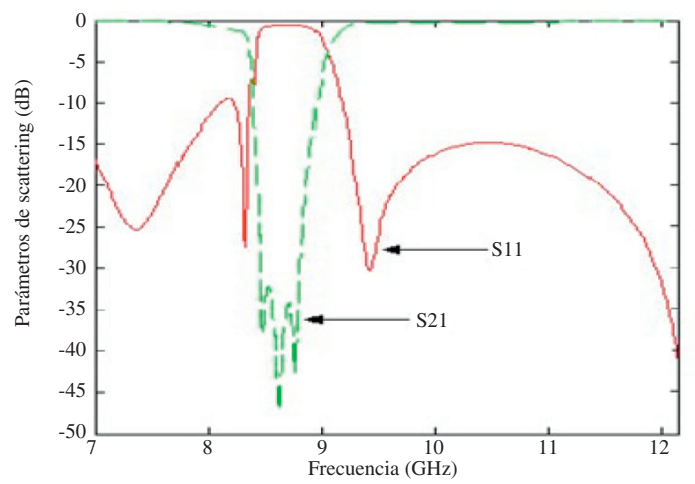

(a)

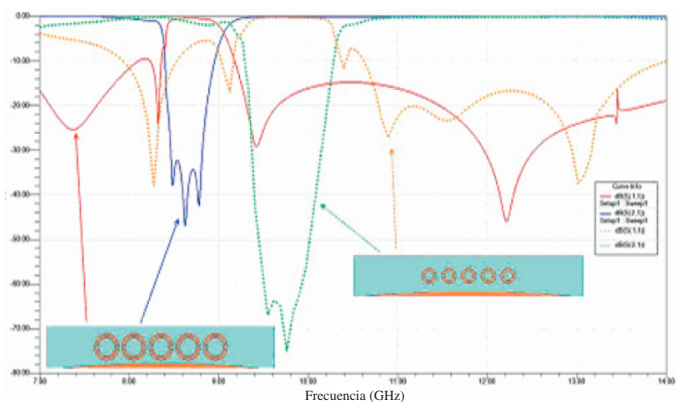

(b)
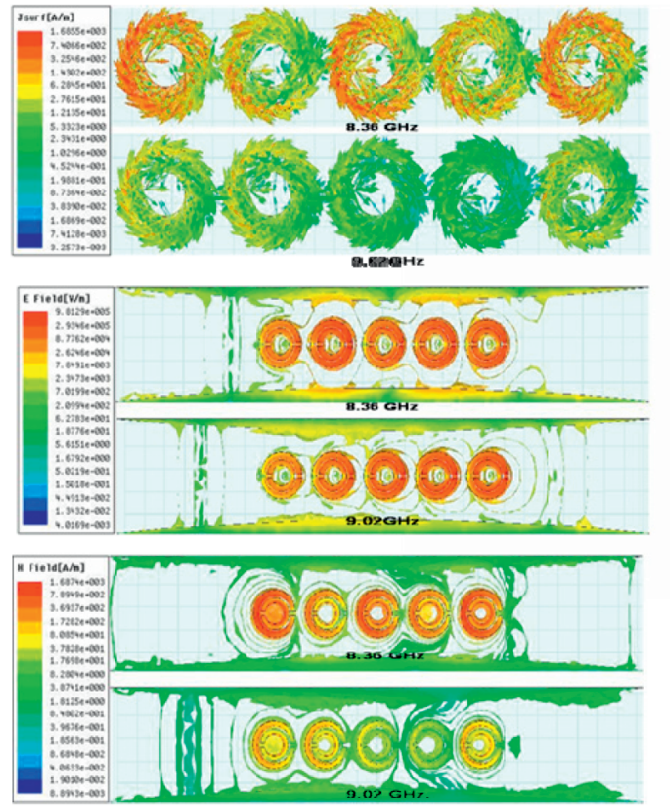

(c)

Figura 13. Magnitud de los parámetros de dispersión en (a) BSF en finline antipodal, (b) dos estructuras diferentes de BSF en finline antipodal, (c) distribución de la densidad corriente en función de la frecuencia. 
de banda y una mayor flexibilidad de diseño. Esta sección describe un filtro finline antipodal supresor de banda, en el cual las dimensiones de los SRRs han sido ajustadas apropiadamente. Los resultados se muestran en la Figura 13. La Figura 13a presenta los parámetros de dispersión y la Figura 13b muestra la variación de la respuesta del filtro al cambiar el radio de los SRRs para demostrar la flexibilidad de este tipo de estructuras. Finalmente, la Figura 13c ilustra el campo electromagnético y la distribución de la densidad de corriente. La Tabla 7 presenta las dimensiones de los resonadores y la Tabla 8 brinda los valores de los parámetros geométricos de la finline antipodal.

Tabla 7. Dimensiones de los resonadores de anillos divididos (SRRs).

\begin{tabular}{|c|c|}
\hline Parámetros & Dimensiones $(\mathbf{m m})$ \\
\hline $\mathrm{r}$ & 2,085 \\
\hline $\mathrm{c}$ & 0,4 \\
\hline $\mathrm{d}$ & 0,3 \\
\hline $\mathrm{g}$ & 0,3 \\
\hline
\end{tabular}

Tabla 8. Dimensiones del filtro finline supresor de banda.

\begin{tabular}{|c|c|}
\hline Parámetros & $\begin{array}{c}\text { Dimensiones } \\
(\mathbf{m m})\end{array}$ \\
\hline Ancho de la guía, $a$ & 22,86 \\
Ancho del sustrato, W1 & 14,2 \\
\hline Longitud total, $\mathrm{L}_{\mathrm{T}}$ & 60 \\
Longitud del filtro, $\mathrm{L}_{l}$, & 53,408 \\
Ancho de la tira de metal, $\mathrm{w}$ & 1,135 \\
Ancho de la metalización, $\mathrm{t}$ & 0,002 \\
Separación entre resonadores, $\mathrm{d}$ & 1,00 \\
\hline & $60 \times 10,16 \times$ \\
Sustrato & 0,51 \\
(L x W x H) & Neltec NX9245 \\
& $\varepsilon_{\mathrm{r}}=2,45$ \\
\hline
\end{tabular}

\section{CONCLUSIONES}

$\mathrm{Al}$ incorporar CSRRs se demostró que es factible producir un filtro que funcione sin rizado en la banda de paso y que produzca a la vez una aguda banda suprimida en la vecindad de su frecuencia de resonancia. Finalmente, después de comparar tanto la información numérica como la experimental, se demostró que el SILPF combinado con los CSRRs es de tamaño compacto y es capaz de proveer una profunda y ultraancha banda suprimida, de gran selectividad y bajo nivel de rizado en la banda de paso. Además, se comprobó con la presencia de SRRs en estructuras finline, que debido a su excelente funcionamiento, pueden utilizarse fácilmente en aplicaciones circuitales que requieran la funcionalidad de filtrado.

\section{AGRADECIMIENTOS}

Los autores agradecen al Ministerio Español de Ciencia e Innovación (MICINN) por el apoyo financiero brindado a través del proyecto TEC200806684-C03-01 y CONSOLIDER-INGENIO 2010 CSD2008-00068.

\section{REFERENCIAS}

[1] J.W. Sheen. "A compact semi-lumped low-pass filter for harmonics and spurious suppression". IEEE Microw. Wireless Compon Lett. Vol. 10, Issue 3, pp. 92-93. March, 2000.

[2] T.M. Lopetegi, A.G. Laso, J. Hernandez, M. Bacaicoa, D. Benito, M.J. Garde, M. Sorolla and M. Guglielmi. "New microstrip "wiggly-line" filters with spurious passband suppression". IEEE Trans. Microw. Theory Tech. Vol. 49, Issue 9, pp. 1593-1598. 2001.

[3] J.T. Kuo, W.H. Hsu and W.T. Huang. "Parallel coupled microstrip filters with suppression of harmonic response". IEEE Microwave Wireless Compon. Lett. Vol. 12, Issue 10, pp. 383-385. 2002.

[4] B.S. Kim, J.W. Lee and M.S. Song. "An implementation of harmonic-suppression microstrip filters with periodic grooves". IEEE Microwave Wireless Compon. Lett. Vol. 14, Issue 9, pp. 413-415. 2004.

[5] F.R. Yang, K.P. Ma, Y. Qian and T. Itoh. "A uniplanar compact photonic-bandgap (UC-PBG) structure and its applications for microwave circuits". IEEE Trans. Microwave Theory Tech. Vol. 47, Issue 8, pp. 1509-1514. 1999.

[6] D. Ahn, J.S. Park, C.S. Kim, Y. Qian, and T. Itoh. "A design of lowpass filter using the novel microstrip defected ground structure". IEEE Trans. Microw. Theory Tech. Vol. 49, Issue 1, pp. 86-93. 2001.

[7] L. Qi, H.M. Salgado, A.M. Moura, and J.R. Pereira. "Dual-band antenna design using an 
EBG artificial magnetic conductor ground plane". IEEE Transactions on Antennas and Propagation Conference, 2008. LAPC 2008. Loughborough, pp. 217-220. 2008.

[8] Q.R. Zheng, B.Q. Lin, Y.Q. Fu and N.C. Yuan. "Characteristics and applications of a novel compact spiral electromagnetic band-gap (EBG) structure". Journal of Electromagnetic Waves and Applications. Vol. 21, Issue 2, pp. 199-213. 2007.

[9] S. Dwari and S. Sanyal. "Compact wide stopband low-pass filter using rectangular patch compact microstrip resonant cell and defected ground structure". Microwave and Optical Technology Letters (MOTL), Interscience Wiley. Vol. 49, Issue 4, pp. 798800. April 2007.

[10] J.B. Pendry, A.J. Holden and D.J. Robbins. "Magnetism from conductors and enhanced nonlinear phenomena". IEEE Trans. Microwave Theory Tech. Vol. 47, Issue 11, pp. 2075-2084. 1999.

[11] P. Gay-Balmaz and O.J.F. Martin. "Electromagnetic resonances in individual and coupled split-ring resonators". Journal of Applied Physics. Vol. 92, Issue 5, pp. 29292936. 2002.

[12] R. Marqués, F. Mesa, J. Martel. and F. Medina. "Comparative analysis of edge and broadside couple split ring resonators for metamaterial design. Theory and Experiment". IEEE Trans. Ant. Propag. Vol. 51, pp. 2572-2581. 2003.

[13] J.D. Baena, J. Bonache, F. Martin, R. Marqués, F. Falcone, T. Lopetegi, M.A.G. Laso, J. García, I Gil and M. Sorolla. "Equivalent circuit models for split ring resonators and complementary split rings resonators coupled to planar transmission lines". IEEE Transactions on Microwave Theory and Techniques. Vol. 53, pp. 1451-1461. 2005.

[14] J. Bonache, F. Marin, F. Falcone, J. Garcia, I. Gil, T. Lopetegi, M.A.G. Laso, R. Marques, F. Medina and M. Sorolla. "Super compact split ring resonators CPW band pass filters". IEEE MTT-S Int. Microwave Symp. Dig., Fort Worth, TX, USA. June, 2004.

[15] J. Bonache, F. Martin and F. Falcone, "Application of complementary split-ring resonators to the design of compact narrow band-pass structures in microstrip technology". Microwave and Optical Technology Letters. Vol. 46, Issue 5, pp. 508-512. 2005.

[16] J. Garcia-Garcia, F. Martin, F. Falcone, J. Bonache, J.D. Baena, I. Gil, E. Amat, T. Lopetegi, M.A.G. Laso, J.A.M. Iturmendi, M. Sorolla, and R. Marques. "Microwave filters with improved stopband based on subwavelength resonators". Microw. Opt. Tech. Lett. Vol. 46, Issue 3, pp. 283-286. 2005.

[17] J. Bonache, F. Martin, J. Garcia-Garcia, I. Gil, R. Marques and M. Sorolla. "Ultra wide band pass filters (UWBPF) based on complementary split ring resonators". Microwave and Optical Technology Letters. Vol. 46, Issue 3, pp. 283-286. 2005.

[18] J.D. Baena, J. Bonache, F. Martin, R. Marques, F. Falcone, T. Lopetegi, M.A.G. Laso, J. Garcia, I. Gil and M. Sorolla. "Equivalent-circuit models for split ring resonators coupled to planar transmission lines". IEEE Trans. Microw. Theory Tech. Vol. 53, Issue 4, pp. 1451-1461. 2005.

[19] F. Arndt, J. Bornemann, D. Grauerholz and R. Vahldieck. "Theory and Design of Low Insertion Loss Fin-Line Filters". IEEE Trans. Microwave Theory Tech. Vol. MTT-30, pp. 155-163. February, 1982.

[20] B. Bhat and S.K. Koul. "Analysis Design and Applications of Fin Lines". Artech House. $1^{\text {st }}$ Ed. Norwood, MA., U.S.A. 1987. ISBN: 0-89006-195-5.

[21] A.M.K. Saad, J.D. Miller, A. Mitha and R. Brown. "Analysis of Antipodal Ridge Waveguide Structure and Application on Extremely Wide Stopband Lowpass Filter". IEEE MTT-S Int. Micr. Symp. Digest, Baltimore, MD, pp. 361-363. June, 1986.

[22] A. Casanueva, A. León, O. González, A. Mediavilla, M. Arias and N. Amar. "Improved Compact Microstrip Low Pass Filter with Novel Distributions of Complementary Split Ring Resonators (CSRRs)". Asia-Pacific Microwave Conference (APMC 2009), pp. 1450-1453. Singapore. December 7-10, 2009. 